\title{
La variabilité du phénotype dans la dystrophie myotonique de Steinert
}

\author{
Jean-Pierre Bouchard
}

\begin{abstract}
RÉSUMÉ: La dystrophie myotonique, aussi connue sous le nom de maladie de Steinert, est une maladie héréditaire qui, lorsque totalement exprimée, présente un tableau clinique caractéristique de dystrophie musculaire accompagné de myotonie et d'anomalies plus ou moins spécifiques de plusieurs autres systèmes. L'apparence physique du patient et certains éléments de son comportement orientent vers le diagnostic que viennent aisément confirmer l'examen neurologique, ophtalmologique et/ou électromyographique. Ce travail rappelle les principaux signes cliniques menant au diagnostic de dystrophie myotonique, insiste sur la variabilité du tableau clinique et présente la forme classique de la maladie. Nous attirons aussi l'attention sur la présence du prominauris dans les formes les plus sévères et les plus précoces. Aucune autre description n'a été faite de cette association, bien que de nombreux auteurs en aient présenté des illustrations fort convaincantes. La reconnaissance de la dystrophie myotonique est essentielle au traitement symptomatique ou préventif de complications telles les arythmies cardiaques, les troubles respiratoires, la cholélithiase, etc., susceptibles de menacer la vie des patients au moment d'une infection ou d'une intervention chirurgicale. Le rôle du médecin est toujours de reconnaître et traiter les maladies et leurs conséquences, même si actuellement tous les yeux sont tournés vers les découvertes plus prestigieuses de la biologie moléculaire.
\end{abstract}

\begin{abstract}
Phenotype Variability in Myotonic Dystrophy Myotonic dystrophy, best known in the French literature as Steinert disease, is a dominantly inherited disorder. When the gene is fully expressed, it shows a characteristic clinical picture. Myopathy and myotonia are the main features, but this is a systemic disease which may involve many other organs such as the central nervous system, eyes, bones, endocrine system, etc. With the complete picture, physical and behavioral features make the diagnosis easy, only to be confirmed by neurological and ophthalmologic examination and/or electromyography. This work reviews the main clinical signs seen in the classical form of the disease. We also want to stress the variability of that picture. Finally, we describe a new dysmorphic sign in myotonic dystrophy: large prominent cupped auricles. Although not recognized, prominauris has been illustrated in a number of publications on myotonic dystrophy. Three of our adult male patients presenting the congenital form of the disease at birth have been investigated to rule out concomitant fragile-X syndrome. Both syndromes include mental retardation and dysmorphic ears. Our patients show the usual testicular atrophy, whereas the fragile-X syndrome includes testicular hypertrophy. Cytogenetic studies were normal. Old clinical description and new physical signs may lack prestige in the perspective of recent molecular biological breakthroughs, but to recognize the disease and help patients live with it will always be the role of practitioners.
\end{abstract}

Can. J. Neurol. Sci. 1989; 16:93-98

\section{Court rappel historique}

La variabilité de l'expression du gène de la dystrophie myotonique de Steinert (DMS) a rendu difficile la description même de la maladie, malgré l'outil clinique exceptionnel qu'est la myotonie. D'abord décrite en 1876 pour la myotonie congénitale par Thomsen qui était lui-même affligée de cette maladie, la myotonie apparaît encore aujourd'hui comme une énigme au point de vue neurophysiologique. Elle se définie cliniquement comme un retard de la relaxation musculaire suite à une contraction soutenue, et elle est surtout apparente après un effort de préhension.

Dans sa monographie sur la maladie de Thomsen, Erb (1886) posait déjà les bases du concept de «l'hétérogénéité génétique» des myotonies en décrivant parmi ses cas de myotonie congénitale des formes distinctes correspondant à la DMS. Ainsi, jusqu'en 1909 on a surtout rapporté des cas de DMS sous l'étiquette de «maladie de Thomsen atypique» ou encore de «myotonie congénitale avec atrophie musculaire».

Les premières descriptions de DMS comme entité distincte ont été rapportées en 1909 sur 9 cas par Steinert et sur cinq cas par Batten et Gibb. ${ }^{1}$ En raison du nombre limité de cas, ces demiers décrivaient la maladie de façon encore équivoque quant au mode de transmission et quant au fait que les hommes semblaient plus souvent touchés que les femmes, par exemple. Plusieurs de ces ambiguités sur les caractéristiques de la maladie sont demeurées dans l'esprit des gens jusque dans les années cinquante.

En 1911 parurent deux autres rapports concernant la présence de cataractes dans la DMS (Greenfield ${ }^{2}$; Kennedy and Obendor ${ }^{3}$ ). Par ailleurs, Curschmann aurait dès 1912 souligné pour la première 
fois la nature extramusculaire et systémique de la maladie en notant, en plus des cataractes, la présence d'atrophie testiculaire et de problèmes mentaux chez certains patients (Harper $\left.{ }^{4}\right)$.

Très tôt on a noté que la plupart des patients mouraient de problèmes cardio-pulmonaires, et ce parfois à la suite de chirurgie. Mais ce qui intriguait le plus les cliniciens ayant décrit la DMS à l'époque, c'était l'apparente sporadicité des cas. En effet, on ne parvenait pas à en observer la transmission de génération en génération de façon aussi claire que pour la myotonie congénitale de Thomsen. En fait, ce n'est que plus tard que l'on a reconnu que l'atteinte plus légère du parent porteur obligatoire était la règle plutôt que l'exception (Harper ${ }^{4}$ ).

C'est ainsi que le mode de transmission demeura incertain jusqu'en 1948, et ceci en bonne partie en raison du «degré extrême de variation de l'expression clinique de la maladie» (Harper $\left.{ }^{4}\right)$. Depuis, on s'est questionné et parfois querellé sur des éléments qui sèment la confusion plutôt que la lumière, tels le concept de «l'anticipation», la transmission exclusivement maternelle de la forme congénitale, etc.

\section{Le tableau clinique classique}

Le phénotype de toute maladie génétique s'exprime généralement de trois façons:

— l'apparence, ou les signes extérieurs;

- la physiologie, qui nous fournit souvent des épreuves diagnostiques et des marqueurs biologiques;

- le comportement.

Les références au tableau clinique classique de la DMS se retrouvent dans une littérature abondante, parsemée d'une plétore d'observations rapportant des déviations multiples de la manifestation typique dans ces trois catégories.

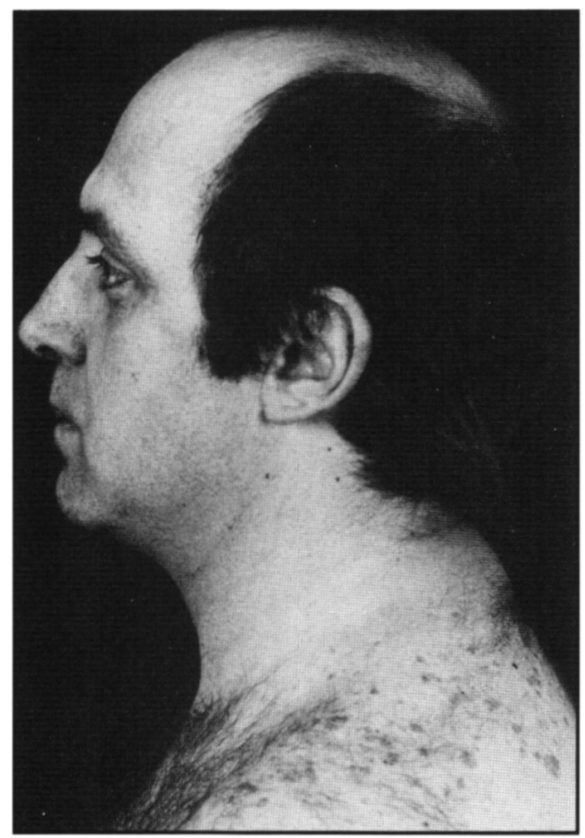

Figure I - Homme de 37 ans, déjà opéré pour cataractes, qui présente une ptose discrète des paupières et un peu de myotonie de préhension. Seul autre signe apparent: une calvitie de type V a (selon la classification de Hamilton, 1951).

\section{L'apparence physique (tête et cou)}

Avec un minimum d'expérience, le diagnostic de DMS peut se faire facilement chez les patients exprimant clairement la maladie. Les signes extérieurs apparaissent principalement au niveau de la tête et du cou, pour ensuite intéresser la partie distale des membres.

Un certain degré de calvitie de type androgénique est fréquemment rencontré chez les hommes atteints (Figure 1) sans qu'on ait pu démontrer une élévation de la testostérone plasmatique. Précoce et sévère chez plus de la moitié des patients mâles, très rare chez les femmes, la calvitie n'est pas associée au niveau d'atteinte musculaire.

Nulle autre maladie ne touche de façon aussi typique les muscles de la mastication. Cette atteinte cause une atrophie fort visible des temporaux, et plus rarement des masséters. Contrairement aux myasthéniques, les patients se plaignent rarement de fatigue masticatoire mais parfois de myotonie, en croquant dans un fruit par exemple. Lorsqu'elle est sévère, la faiblesse entraine une mâchoire tombante et une bouche béante.

Habituellement associée à la faiblesse des muscles faciaux, la ptose des paupières supérieures est partie intégrante du tableau classique. Le releveur de la paupière est pratiquement le seul muscle extraoculaire à être touché, et il est parfois compensé, au début, par une rétraction de la partie externe du muscle frontal. La ptose est très souvent asymétrique, et parfois exagérée en apparence par de l'énophtalmie.

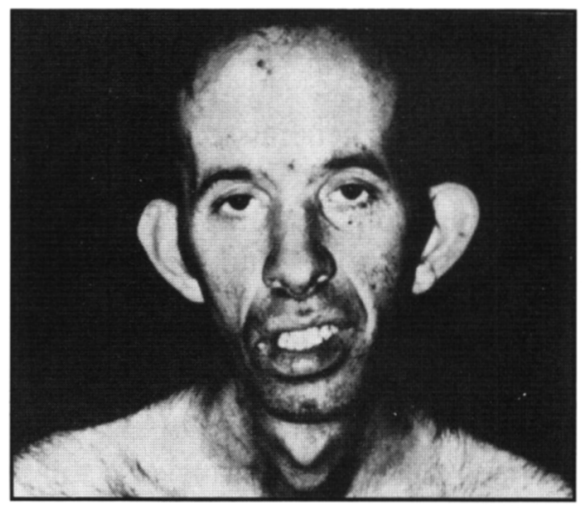

Figure 2 - Faciès typique de la dystrophie myotonique: "the long, thin face with temporal and masseter wasting and frontal balding is characteristic" (Brooke 7 ; reproduction avec permission de l'auteur et de l'éditeur). Noter ici les grandes oreilles décollées; plusieurs auteurs ont déjà illustré ce phénomène, sans le souligner.

En analysant les photographies présentées dans la littérature de même qu'en observant nos propres patients, nous avons été frappés par la présence de cas présentant de grandes oreilles décollées et parfois mal ourlées parmi les patients mâles atteints d'une forme sévère et précoce de DMS. Nous croyons qu'il s'agit ici d'un signe mineur, mais authentique, présent chez moins de $10 \%$ des patients mâles, et que la littérature neurologique a très souvent illustré (figure 2) sans pour autant en faire mention (Merritt ${ }^{5}$; Schotland ${ }^{6}$; Harper ${ }^{4}, 9$; Brooke ${ }^{7}$ ). Quatre de nos patients porteurs d'une forme congénitale présentent cette anomalie, et deux d'entre eux ont subi une intervention chirurgicale pour correction de prominauris. Il n'est pas sûr que tous les cas illustrés ont la forme congénitale de la 


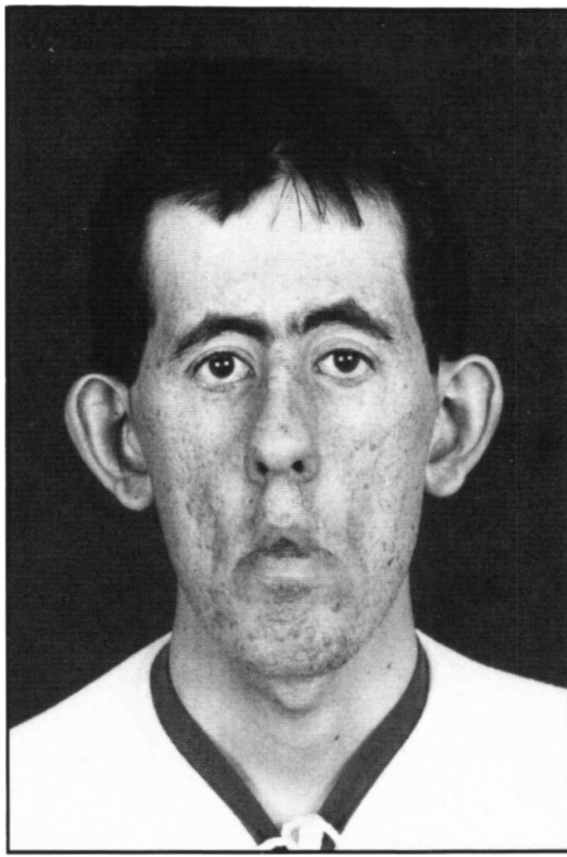

Figure 3A-Homme de 23 ans; forme congénitale de DMS. Noter le faciès inexpressif. la légère ptose asymétrique, l'atrophie des muscles temporaux et des masseters, la bouche en forme de tente, l'hypotélorisme et le prominauris que nous proposons comme nouveau signe dysmorphique.

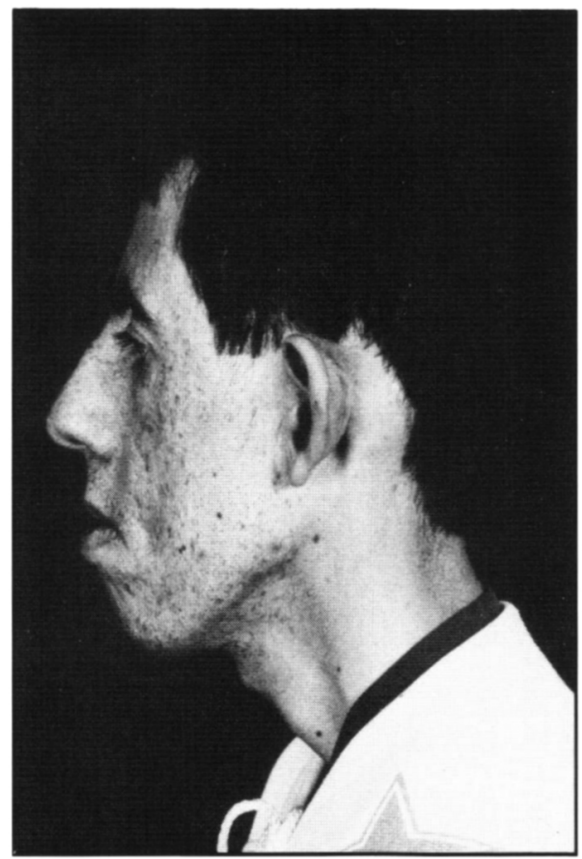

Figure 3B-Même patient présentant un profil «simiesque». Les os de la face semblent aplatis, la mastoïde proéminente et le pavillon de l'oreille est orienté de façon coronale.

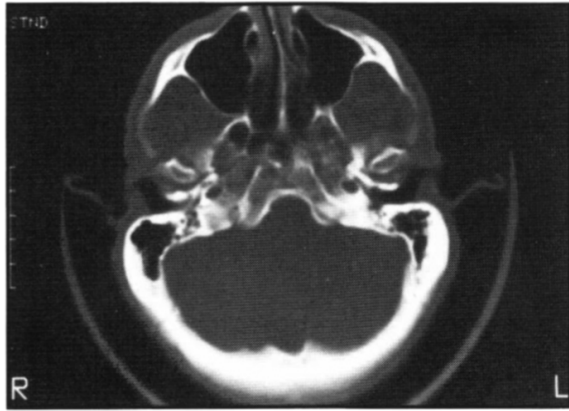

Figure $3 C-L a$ tomographie axiale révèle que la mensuration bi-mastö̈dienne est augmentée par rapport à la bi-malaire yui est normale. On note aussi une orientation anormale du conduit auditif externe: d'abord fortement orienté vers l'arrière par le tragus, il se dirige ensuite nettement trop vers l'arrière dans sa portion ossense. Le cerveau et les sinus ne présentent pas d'anomalie significative.
DMS, mais au moins deux autres cas sont présentés comme tels dans la monographie de Harper $^{4}$ (pages 20 et 21).

Une analyse du karyotype a été effectuée pour trois de ces jeunes adultes afin d'éliminer un syndrome de l'X fragile, entité où l'on rencontre aussi un retard mental et de grandes oreilles chez les mâles. ${ }^{8}$ Chez les porteurs de ce syndrome on trouve également une hyperorchidie, ce qui n'est évidemment pas le cas chez nos patients qui présentent plutôt de l'atrophie testiculaire et chez qui l'X s'est avéré normal. La figure 3 illustre bien le prominauris, la bouche «en forme de tente» et les déformations faciales chez un patient retardé âgé de 23 ans et porteur d'une forme congénitale de DMS. Son frère de 26 ans, opéré pour pieds bots à l'âge de deux ans, est moins retardé mais présente tout de même des oreilles d'apparence anormale (figure 4).

Seul signe audible, la voix nasillarde fait presque toujours partie du tableau classique. Elle peut aussi apparaître précocement dans le syndrome. D'est surtout l'atteinte du palais mou qui en est responsable, mais la faiblesse des cordes vocales contribue aussi parfois au caractère enrouée de la voix. La langue peut participer aux troubles de la parole et de la déglutition en raison de faiblesse et d'atrophie, plus rarement de myotonie. L'atteinte des principaux muscles pharyngés et de la partie supérieure de l'oesophage est souvent responsable d'une dysphagie plus ou moins importante, parfois exagérée par la myotonie causée par l'ingestion d'aliments froids.

Enfin, l'atrophie des muscles sterno-cléido-mastoïdiens est fréquente et donne au cou un aspect décharné. Les patients éprou- vent alors de la difficulté à lever la tête de l'oreiller. Il peut aussi y avoir une faiblesse marquée des extenseurs du cou, ce qui entraine tardivement une flexion antérieure constante de la tête.

\section{Le tronc et les membres}

La DMS est une dystrophie axiale et distale. Elle préserve le plus souvent et très longtemps la force de la musculature proximale. La scoliose est rare, mais le thorax aplati et l'amplitude respiratoire faible sont courants. Il n'y a pas d'hyperlordose comme dans la dystrophie de Duchenne ou celle de Landouzy-Déjerine, mais on note souvent un faiblesse de la paroi abdominale laissant apparaître un "petit bedon», même chez les patients émaciés. Quand à l'atrophie testiculaire, elle est encore inexpliquée. Souvent asymétrique, elle est présente chez environ $70 \%$ des patients mâles tout en leur permettant une fertilité à peu près normale.

Au niveau des membres, l'atteinte est d'emblée distale. La faiblesse et l'atrophie progressives surtout du compartiment antérieur de la jambe donnent des pieds tombants et une démarche «à claquette». Elles touchent également la loge cubitale de l'avant-bras (fléchisseurs du poignet et des doigts), région qui est aussi la plus affectée par la myotonie de préhension. Les extenseurs des doigts sont eux aussi souvent très faibles, quoique moins atrophiques, et on peut y démontrer la myotonie de percussion tout aussi bien qu'au niveau du court abducteur du pouce. A mesure que la faiblesse devient plus importante, on assiste souvent à une régression de la myotonie. L'atteinte musculaire surtout distale et le 


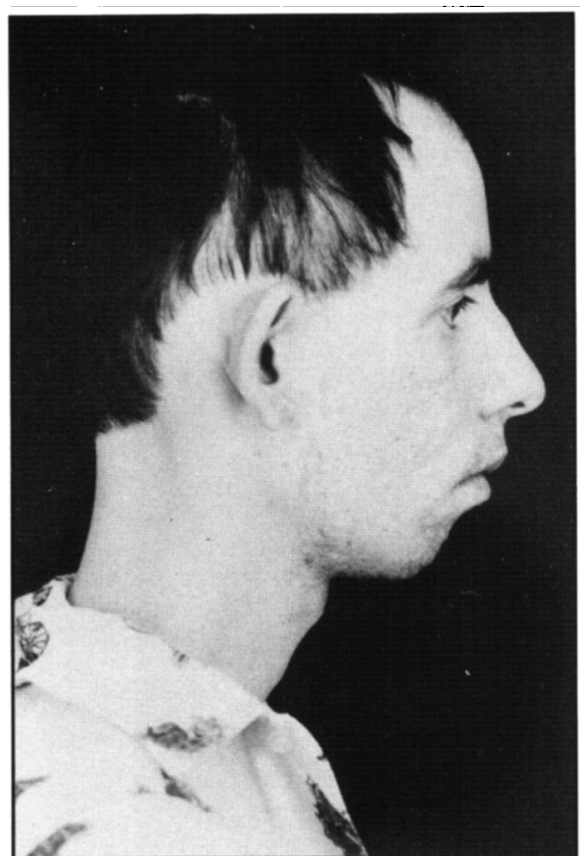

Figure 4-Cet homme de 26 ans, frère du patient de la figure 3, a été opéré pour des pieds bots à l'age de deux ans. Il présente plusieurs autres signes dysmorphiques: palais ogival, rétrognathie et prominauris bilatéral. Les oreilles n'ont pas d'anthélix et leur hauteur est de $66 \mathrm{~mm}$ à droite et 67 mm à gauche (75 et 80ième percentile).

Tableau 1: Signes éxtérieurs: tête et cou

Calvitie

Atrophie temporale

Faiblesse des muscles faciaux (perte d'expression)

Ptose palpébrale

Enophtalmie

Oreilles décollées (grandes et mal ourlées)

Bouche entrouverté

Voix nasillarde

Atrophie des sterno-cleido-mastoïdiens

phénomène encore mal compris de la myotonie, parfois assimilée aux crampes, spasmes, décharges de «hautes fréquences», etc., alimentent encore aujourd'hui des hypothèses neurogéniques pour tenter d'expliquer la maladie.

\section{Les «marqueurs physiologiques»}

Dans cet article qui se veut surtout clinique, nous ne parlerons que des tests courants en neuromusculaire. Le dosage de la créatine-kinase (CPK) n'est d'aucune utilité pour le diagnostic ou le pronostic de la maladie; à ce jour aucun autre test sanguin en usage dans nos laboratoires hospitaliers ne peut servir à ajouter de la précision. L'hyperglycémie provoquée est fréquemment anormale, mais le diabète clinique est rare.

La biopsie musculaire n'est pas nécessaire au diagnostic. Les nombreuses anomalies morphologiques retrouvées à la biopsie ne sont pas spécifiques prises une à une, mais l'ensemble forme un tableau très suggestif de la maladie. En l'absence presque totale de nécrose, on retrouve un grand nombre de noyaux centraux et
Tableau 2: Signes éxtérieurs: tronc et membres

Respiration superficielle

Relachement de la paroi abdominale (petit bedon)

Atrôphie testiculaire

Myotonie de préhension et de percussion

Faiblesse et atrophie des muscles distaux:

- Fléchisseurs (et extenseurs) du poignet et des doigts

(faiblesse de la préhension)

-Dorsifléchisseurs des pieds

(pieds tombants et démarche «à claquette»)

Tableau 3: Marqueurs physiologiques

Électromyographie:

- Myotonie électrique

- Tracé myopathique

Biopsie musculaire

- Hypertrophie des fibres de Type II

- Atrophie des fibres de Type I

Cataractes

Troubles du rythme cardiaque

Tableau 4: Manifestations oculaires

Cataractes

Dégénérescence rétinienne (rare)

Myotonie: diplopie (rare); «lid-lag»; blépharospasme

Faiblesse: ptose; malocclusion des paupières (kératite)

Basse pression intra-oculaire et énophtalmie

\section{Tableau 5: Troubles du comportement}

Le patient nie ou minimise les symptomes,

Est peu actif,

Parfois hypersomniaque,

Et peut présenter une atteinte mentale

(sévère dans la forme congénitale)

plusieurs changements architecturaux tels les masses sarcoplasmiques et les «ringbinden». L'histochimie montre une hypertrophie des fibres de type II au stade plus précoce et surtout myotonique, alors que l'atrophie est toujours plus marquée pour les fibres de type I. Rappelons la présence occasionnelle de quelques fibres musculaires dites «ragged-red» ou «moth-eaten». La microscopie électronique n'apporte aucun renseignement supplémentaire pour le diagnostic.

L'électromyographie est considéré à juste titre comme une aide précieuse lorsque le tableau clinique ne permet pas de poser d'emblée le diagnostic de DMS. La présence de bouffées de potentiels de très courte durée et de fréquence élevée lors de l'insertion de l'aiguille, après percussion du muscle étudié ou après le mouvement, constitue la description de la myotonie électrique, et peut être retrouvée même chez des patients asymptomatiques. La fréquence variable au cours de la même bouffée produit un son caractéristique à l'audio («dive bomber»). Un tel phénomène peut aussi se rencontrer dans les autres maladies myotoniques et dans d'autres troubles neuromusculaires, mais il n'est pas alors associé à un tracé myopathique lors de la contraction volontaire comme c'est le cas dans la DMS. Ces anomalies de l'électromyogramme sont présen- 


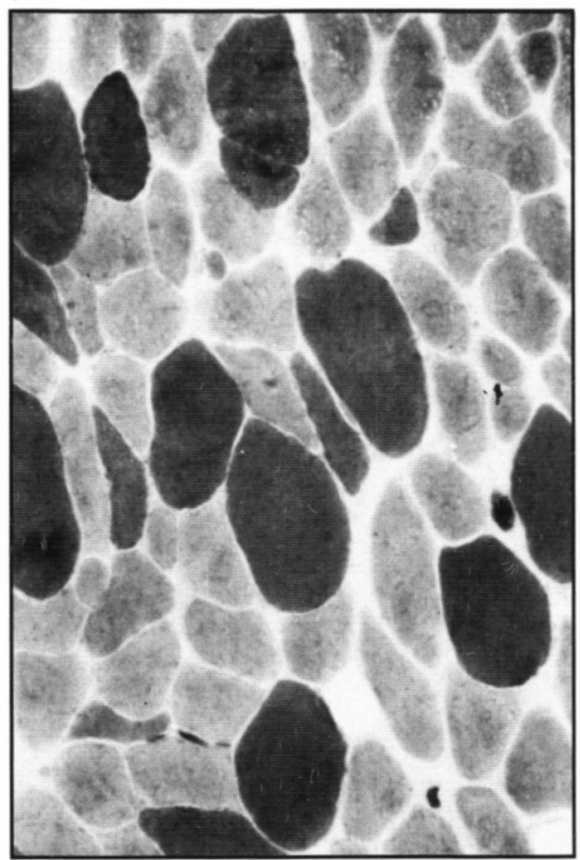

Figure 5 - Femme, 33 ans, biopsie du gas. trocnemius (ATPase, $p H$ 9.4, $x$ 100). Hypertrophie nette des fibres musculaires de type II et atrophie tout aussi évidente des fibres de type l. Cette distribution des fibres est assez typique de la maladie, surtout à ses débuts.

tent dans pratiquement tous les muscles, y compris le diaphragme, mais en pratique les muscles les plus souvent étudiés sont l'abductor pollicis brevis et l'extensor digitorum communis.

Un autre élément très utile pour confirmer le diagnostic est l'histoire familiale et/ou la présence de cataractes dont les caractères spéciaux à l'examen avec une lampe à fente peuvent suggérer à eux seuls le diagnostic de DMS (voir Mathieu J et al., même numéro). A partir des petites opacités colorées sous-capsulaires postérieures, la confluence des lésions provoquent souvent avec le temps une cataracte mature qu'il faut extirper chirurgicalement. Les autres manifestations oculaires de la DMS ne sont pas spécifiques et peuvent être associeés à la myotonie (diplopie, «lid-lag»), à la faiblesse (ptose, non occlusion palpébrale causant des kératites) ou encore à l'atteinte du système nerveux autonome (hypotension intra-oculaire causant l'énophtalmie) (Tableau 4).

Presques tous les systèmes sont touchés par la DMS, mais de façon diverses et peu spécifiques (cardiaque, respiratoire, endocrinien, digestif, système nerveux central). Au niveau cardiaque, les troubles du rythme viennent en tout premier lieu, causant parfois des syncopes ou une mort subite comme chez deux de nos patients dans la vingtaine.

L'hypoventilation chronique fait partie du tableau classique de la DMS. De plus, une toux non efficace et des micro-aspirations contribuent à l'embarras bronchique et à l'incidence augmenté de pneumonie. La respiration est courte et superficielle. Souvent le patient doit reprendre son souffle après s'être déplacé ou tout simplement exprimé. Mais la condition la plus dramatique survient habituellement après une intervention chirurgicale, surtout à l'étage supérieur de l'abdomen, alors que le retour à la respiration spontanée post-curarisation peut prendre des jours et même des semaines. L'histoire familiale révèle souvent des parents porteurs de DMS qui sont décédés dans de telles conditions il y a quelques décades, sans que le diagnostic ait été porté.

Du côté endocrinien, les problèmes semblent plus apparents que réels. Nous avond déjà souligné le peu de diabète clinique même si plus de $50 \%$ des patients présentent des anomalies à l'hyperglycémie provoquée. Il en est de même chez les patients mâles chez qui l'atrophie testiculaire ne se traduit jamais par une infertilite, et qui ont des taux normaux de testostérone. Les femmes ont aussi une fertilité normale, mais le produit de la grossesse est souvent anormal (voir Paris $\mathrm{G}$ et al, même numéro).

Du côté digestif, les symptômes sont inconstants et souvent imprécis, allant de la dysphagie haute à la constipation et aux troubles sphinctériens, en passant par les régurgitations, la flatulence, la cholélithiase, etc.

\section{Le comportement}

Enfin, le comportement dans la DMS est bien particulier. Chez l'adulte, il faut dire que le faciès parfois lugubre et la voix trainante et nasillarde donnent une impression souvent non vérifée de débilité mentale. De plus les patients sont souvent hypoactifs et parfois hypersomniaques. Il s'agit là parfois d'un lourd handicap que l'on peut traiter comme la narcolepsie. Mais ce qui les caractérise le mieux, c'est un naturel renfermé et parfois méfiant, la négation ou la minimisation des symptômes (qui rendent ardue l'histoire naturelle de la maladie) et le peu de fidélité aux conseils et traitements.

\section{La variabilité du phénotype}

Plusieurs auteurs, et en particulier Harper ${ }^{4.9}$ se sont attardés à définir le pourcentage de patients présentant l'un ou l'autre des symptômes et des signes ici décrits. Bien que utiles pour mieux comprendre le spectre de la maladie, ces statistiques apportent peu au diagnostic d'un cas individuel. A un moment ou l'autre de l'évolution, et selon le degré de sophistication de l'examinateur, ces données changent considérablement. Le tableau va du patient asymptômatique (forme partielle de DMS, ou latence de l'expressivité du gène?) aux formes précoces et sévères (forme congénitale; forme infantile?; forme jeune?) en passant par toutes les possibilités d'expression phénotypique que permettent la présence de tant de variables. Ces études seront bientôt complétées par l'utilisation de marqueurs génétiques, et sans doute pourrons nous comprendre un jour les causes d'une si extraordinaire variation. Celle-ci ne semble même pas indiquer une hétérogénéité génétique puisque toutes les variantes peuvent se retrouver dans la même fratrie.

Quant au prominauris, nous nous proposons d'évaluer la fréquence relative et la pathogénie de cette malformation dans les formes précoces et sévères de la DMS.

\section{REMERCIEMENTS}

Nous remercions le Dr. Rachel Laframboise (Service de médecine génétique, CHUL) pour son évaluation des patients présentant une forme congénitale de dystrophie myotonique, et le Dr. Réginald Langelier (Département de radiologie, Hôpital de l'Enfant-Jésus) pour son étude attentive des signes radiologiques.

\section{RÉFÉRENCES}

1. Batten FE, Gibb HP. Myotonia atrophica. Brain 1909; 32: 187-205.

2. Greenfield JG. Notes on a family of «myotonia atrophica» and early cataract, with a report on an additional case of myotonia atrophica. Rev Neur Psych (Edin) 1911; 9: 169-181. 
3. Kennedy F, Obendorf CP. Myotonia atrophica, with a report of two cases. JAMA 1911; 57: 1117-1118.

4. Harper PS. Myotonic Dystrophy. In: «Major Problems in Neurology», vol. 9 Philadelphia: W B Saunders Co. 1979.

5. Merritt HH. A Textbook of Neurology (Fourth edition). Philadephia: Lea \& Febiger 1967.

6. Schotland DL. Muscular Dystrophy. New York: Medcom 1971.
7. Brooke MH. A Clinician's View of Neuromuscular Diseases (Second edition). Baltimore: Williams \& Wilkins 1986.

8. Hagerman RH, McBogg P. The Fragile X Syndrome. Dillon (Colorado): Spectra Publishing Co. 1983.

9. Harper PS. Myotonic disorders. In: Engel AE, Banker BQ, eds. Myology, vol. 2, chapter 40. New York: McGraw-Hill 1986. 